\title{
Saúde mental em tempos de pandemia da COVID-19: uma revisão de literatura
}

\author{
Mental health in times of the COVID-19 pandemic: a literature review
}

Salud mental em tempos de la pandemia COVID-9: uma revisión de la literatura

Raquel do Carmo Hubner Moreira ${ }^{1 *}$, Maria Gabriela Elias d'Assumpção', Aline Ferreira de Souza', Camila Moreira Costa ${ }^{1}$, Gabriela Roque Pereira ${ }^{1}$, Isabela Guedes Paiva ${ }^{1}$, Mabeli Ribeiro da Mata ${ }^{1}$, Paula Moreira Sena1', Victor Gonçalves Miranda1', Maurício Lacerda Caldeira Filho'.

\section{RESUMO}

Objetivo: Discutir a saúde mental no contexto da pandemia da doença do Coronavírus 2019 (COVID-19). Revisão bibliográfica: diante da pandemia causada pela COVID-19, declarada em março de 2020, pela Organização Mundial da Saúde, vários estudos vêm demonstrando um maior risco de desenvolvimento de síndromes psiquiátricas nos pacientes com COVID-19, bem como nos profissionais da saúde da "linha de frente". As causas propostas incluem alterações neurológicas, consequentes à infecção pelo vírus e o estresse gerado pela situação pandêmica, como as medidas de isolamento social adotadas, o receio de transmitir a doença inadvertidamente para amigos e familiares e as perdas financeiras. Outro fator considerado importante é a sobrecarga de trabalho com a grande quantidade de pacientes e perda de médicos e enfermeiros por morte ou afastamento por doença. Considerações finais: É imprescindível a estruturação de uma rede de apoio psicológico e psiquiátrico para os indivíduos mais afetados pela pandemia, de modo a possibilitar intervenções precoces, se necessárias.

Palavras-chave: COVID-19, Saúde mental, Assistência psicológica, Psiquiatria.

\begin{abstract}
Objective: This article intends to discuss mental wellness within the context of the Coronavirus (COVID-19) pandemic. Bibliographic review: In view of the pandemic caused by COVID-19, declared in march 2020, by the World Health Organization, several studies have shown an a increased risk of developing psychiatric syndromes in patients with COVID-19 and the "frontline" health care workers. The proposed causes include neurological changes induced by the viral infection and the stress generated by the pandemic, such as the restrictive social isolation measures adopted, fear of unintentionally spreading the virus to family and friends and financial losses. Another important factor is work overload, with a large number of patients and the loss of physicians and nurses due to death or sick leave. Final considerations: It is essential to build a psychological support network and access to psychiatric services for these groups to allow for an early intervention if necessary.
\end{abstract}

Keywords: COVID-19, Mental health, Psychological assistance, Psychiatry.

\section{RESUMEN}

Objetivo: Este artículo busca discutir la salud mental en el contexto de la pandemia de enfermedad por Coronavirus de 2019 (COVID-19). Revisión bibliográfica: Ante la pandemia provocada por COVID-19, declarada en marzo de 2020, por la Organización Mundial de la Salud, varios estudios han mostrado un mayor riesgo de desarrollar síndromes psiquiátricos en pacientes con COVID-19, así como en profesionales de la

${ }^{1}$ Instituto Metropolitano de Ensino Superior (IMES), Ipatinga - MG. *E-mail: quelhubner@hotmail.com 
salud de la "primera línea". Entre las causas propuestas se encuentran las alteraciones neurológicas derivadas de la infección por el virus y el estrés generado por la situación pandémica, como las medidas de aislamiento social adoptadas, el miedo a transmitir inadvertidamente la enfermedad a amigos y familiares, y pérdidas económicas. Otro factor que se considera importante es la carga de trabajo con la gran cantidad de pacientes y la pérdida de médicos y enfermeras por fallecimiento o baja laboral. Consideraciones finales: Es fundamental estructurar una red de apoyo psicológico y psiquiátrico para las personas más afectadas por la pandemia, con el fin de posibilitar intervenciones tempranas, si es necesario.

Palabras clave: COVID-19, Salud mental, Asistencia psicológica, Psiquiatría.

\section{INTRODUÇÃO}

Os primeiros casos da doença do coronavírus 2019 (COVID-19) foram identificados na cidade de Wuhan, na China, em dezembro de 2019. No final de janeiro de 2020, a Organização Mundial da Saúde (OMS) anunciou uma emergência de saúde pública e em março de 2020 declarou o surto do Coronavírus 2 da Síndrome Respiratória Aguda Grave (SARS-CoV-2) como uma pandemia. A COVID-19 se espalhou rapidamente ao redor do globo e sobrecarrega os sistemas de saúde, gerando diversos desafios, como a questão da alocação de recursos escassos. Ademais, houve a necessidade de promover o isolamento social e lockdowns, com possíveis impactos sobre a saúde mental da população (ZHU N, et al., 2020; LI Y, et al., 2021; NOCHAIWONG S, et al., 2021).

Sabe-se que a COVID-19 é uma afecção infectocontagiosa viral que pode variar em suas manifestações, desde sintomas leves, como febre, tosse e mialgia até quadros mais graves e desfechos como o óbito do paciente. Os sintomas mais referidos são associados ao trato respiratório, no entanto, tem-se observado que essa patologia tem caráter multissistêmico, afetando inclusive o cérebro, de modo a acarretar repercussões neurológicas, e em alguns casos o paciente pode apresentar sequelas cognitivas a longo prazo (FIORILLO A e GORWOOD P, 2020; SALARI N, et al., 2020).

Outrossim, o SARS-CoV-2 é um vírus com maior potencial de infecção e alta taxa de transmissão comparado aos outros de seu grupo, como o SARS-CoV-1. Como a transmissão do vírus se dá através de gotículas de secreção das vias respiratórias do indivíduo contaminado para o saudável, a disseminação tomou grandes proporções. Com isso, a pandemia da COVID-19 provocou grandes mudanças no estilo de vida das pessoas, assim como afetou o bem-estar mental e físico, comprometendo a saúde, a liberdade e a condição econômica (CASTALDELLI-MAIA JM, et al., 2021; SANTABÁRBARA J, et al., 2021).

Diante do aumento no número de infecções e de óbitos decorrente de um vírus altamente contagioso e com poder de mortalidade elevado, alguns países instituíram, em sua população, a chamada quarentena e o distanciamento social, por períodos indefinidos, de modo a mitigar a disseminação viral. Ainda que tais medidas restritivas possam ser eficazes na contenção do SARS-CoV-2, estas podem acarretar sofrimento psicossocial, com desfechos emocionais negativos como estresse, ansiedade e depressão (PFEFFERBAUM B e NORTH CS, 2020).

Consequentemente a isso, surge-se uma preocupação do impacto da pandemia sobre o bem-estar psicológico da população, uma vez que o contexto social, econômico e sanitário predispõe maior risco de desenvolvimento de síndromes psiquiátricas, como ansiedade, depressão, estresse e insônia. Esses transtornos podem ser desencadeados por experiências negativas, em decorrência da doença, da restrição da liberdade pessoal, de mudanças repentinas, da impossibilidade de planejamento futuro e do distanciamento social, associada à preocupação com a própria saúde, de parentes e de conhecidos, bem como das grandes perdas financeiras inesperadas (ABDALLA SM, et al., 2021).

Destarte, este trabalho teve por objetivo realizar e descrever uma revisão integrativa de estudos existentes na literatura médica e publicados após o início da pandemia acerca do impacto da COVID-19 sobre a saúde mental da população. Ademais, objetivou-se descrever quais as síndromes mais comuns e as medidas propostas e/ou adotadas para prevenção e tratamento de transtornos psiquiátricos oriundos direta ou indiretamente da pandemia. 


\section{REVISÃO BIBLIOGRÁFICA}

\section{Conceito}

De acordo com a OMS, saúde mental é uma virtude, na qual o indivíduo desenvolve habilidades pessoais a fim de lidar com as adversidades da vida e encontrar-se apto a conviver em sociedade. Dessa forma, a saúde mental não se resume somente à ausência de doença, mas também à capacidade do indivíduo em viver e interagir com seus semelhantes, e ela é considerada fundamental à saúde como um todo (WORLD HEALTH ORGANIZATION (WHO), 2018).

A saúde mental é influenciada por diversos fatores biopsicossociais. Em relação aos determinantes sociais, é sabido que o abandono afetivo na infância e a privação, seja ela material, emocional ou relacionada à violência, têm um peso importante no desenvolvimento futuro de transtornos psiquiátricos. Na vida adulta, soma-se a essas questões, o estresse cotidiano com o trabalho e com a pandemia da COVID-19, os receios e os temores suscitados pela doença atuam como agravantes do bem-estar psicológico (PATEL V, 2018; ANAND V, et al., 2021).

Analogamente, a American Psychiatric Association (APA) define transtorno psiquiátrico não como ausência de bem-estar mental, mas como uma alteração significativa das emoções, capacidade de pensamento ou comportamento e que gera desconforto ou problemas para o indivíduo quando interage em atividades sociais, de trabalho e no ambiente familiar. Há um espectro, desde quadros mais leves, como fobias específicas, até situações que exijam a internação hospitalar do paciente para tratamento adequado (PAREKH R, 2018).

\section{Etiologia}

Vários fatores podem estar envolvidos no surgimento de transtornos mentais no cenário da COVID-19. Entre eles, o medo inerente à pandemia, efeitos adversos de tratamentos, estresse financeiro, isolamento social e até a própria fisiopatologia do vírus. Além de tudo, os estudos revelaram uma grande angústia e ansiedade devido à infecção pelo SARS-CoV-2, que resultou em um baixo bem-estar psicológico, aumento nas tendências suicidas e piora de condições pré-existentes da saúde mental. Ademais, nota-se que afetou gravemente as relações familiares e a dinâmica social por meio do aumento nos casos de violência doméstica, e uso abusivo de álcool (ASMUNDSON G, et al., 2020; BUTTELL F e FERREIRA R, 2020; CLAY J e PARKER M, 2020; SALADINO V, et al., 2020; SHER L, 2020; PIETROMONACO P e OVERALL N, 2021).

Percebe-se que o estresse do isolamento social induz uma desregulação do sistema neuro-imuneendócrino. Este mecanismo tem-se mostrado vital para o desenvolvimento de distúrbios psicológicos, haja visto que a privação de interação social pode estimular o eixo Hipotálamo-Pituitária-Adrenal (HPA), que aumenta a secreção de glicocorticoides, notadamente o cortisol, o qual é o hormônio associado ao estresse. Nesse contexto, pode haver a inibição de secreção dos chamados "hormônios sociais", como a vasopressina e a ocitocina, pelo hipotálamo, que amplifica o efeito negativo do estímulo inicial (WANG L, et al., 2020).

Outrossim, uma das relações sugeridas entre a doença e os desfechos negativos resultantes sobre a saúde mental é pelo envolvimento de citocinas, as quais são pequenas proteínas que atuam como moduladores de funções celulares. Nessa circunstância, as concentrações elevadas dessas proteínas são observadas em outros transtornos psiquiátricos, de maneira similar à COVID-19, e postula-se que elas interferem na síntese, secreção e reabsorção de diversos neurotransmissores no sistema nervoso central, como as monoaminas. Nessa conjuntura, essas alterações estão relacionadas à fisiopatologia de transtornos como a ansiedade, depressão e transtornos obsessivo-compulsivos (RAONY I, et al., 2020).

Portanto, é possível inferir que a infecção pelo SARS-CoV-2 provoca alterações no sistema nervoso central e predispõe o desenvolvimento de sinais e sintomas psicológicos e psiquiátricos no paciente. Nessa perspectiva, essas mudanças podem ocorrer tanto sob influência direta do vírus, por meio da reação imunológica e aumento de citocinas, quanto pelo estresse com a pandemia da COVID-19, o medo de contrair a doença e propagá-la inadvertidamente para amigos e familiares e o isolamento social (ASMUNDSON G, et al., 2020; WANG L, et al., 2020). 


\section{Os impactos mentais gerados pela pandemia}

Sabe-se que a pandemia do COVID-19 representa, além de uma grande crise de saúde pública e econômica, um impacto substancial à saúde mental. Embora haja uma escassez de evidências atuais sobre os efeitos diretos da doença em relação à saúde mental, se infere as consequências negativas que atingem a comunidade de uma forma geral. Para efeito comparativo, na epidemia de SARS-CoV-1, ocorrida entre 2002 e 2004, foi constatada a presença de manifestações psiquiátricos na sociedade geral, que meses após o controle da doença, e dentre os pacientes que contraíram o vírus, se observou maior prevalência de sintomas depressivos (VINDEGAARD N e BENROS ME, 2020; LEE Y, et al., 2021).

Além disso, a imposição do isolamento social por tempo indeterminado, a rápida propagação e o elevado número de mortes causados pela doença, bem como as crescentes perdas financeiras que atingiram muitos indivíduos, são alguns exemplos de repercussões do atual quadro situacional ao povo e representam o risco elevado de transtornos psiquiátricos em toda a sociedade. Nessa situação, o aumento da solidão e a diminuição das interações sociais, em particular, são fatores de risco bem conhecidos para vários transtornos mentais, sobretudo esquizofrenia e depressão maior. Além do mais, o aumento de angústia ou Transtornos de Estresse Pós-Traumático (TEPT) e de sintomas de ansiedade também foram notados (FIORILLO A e GORWOOD P, 2020; SANTABÁRBARA J, et al., 2021).

Nochaiwong S, et al. (2021) observou que, nas pessoas em geral, o predomínio global combinado de problemas de saúde mental durante a pandemia é maior que o pré-pandêmico, que considerou, também, as consequências psicossociais. Nesse panorama, foi notada uma série de problemas, como ansiedade, depressão, TEPT, estresse, sofrimento psicológico e problemas com sono. Já em relação aos pacientes com COVID-19 em curso, estudos realizados por Deng J, et al. (2021) sugerem que cerca de $45 \%$ apresentam depressão, $47 \%$ dos pacientes apresentam ansiedade e $34 \%$ apresentam distúrbios do sono, demonstrando um aumento substancial em relação ao grupo populacional.

Devido aos inúmeros obstáculos encontrados durante o enfrentamento à COVID-19, como os receios pela segurança pessoal, a carga excessiva de trabalho, uma rede de apoio limitada, dentre outros fatores concomitantes, os profissionais de saúde se tornaram mais vulneráveis ao desenvolvimento de transtornos psiquiátricos e psicológicos. Dessa forma, a prevalência de ansiedade, TEPT, depressão moderada e má qualidade de sono entre indivíduos que atuam na área da saúde durante a COVID-19 tem altas estimativas e, quando comparada à população geral, se mostra maior (GOLD JA, 2020; PAPPA S, et al., 2020; LI Y, et al., 2021).

Em pacientes que apresentam transtornos psiquiátricos ou sofrimentos psicológicos pré-existentes, têm uma suscetibilidade à piora dos seus sintomas durante a pandemia. Dessa forma, Vindegaard $\mathrm{N}$ e Benros $\mathrm{ME}$ (2020) demonstraram que, dos pacientes com transtornos alimentares prévios, 37,5\% tiveram piora da sintomatologia e 56,7\% apresentaram sintomas adicionais de ansiedade. Aliás, Xiang YT, et al. (2020) apontou que, no contexto da China, as restrições de movimento e isolamento social, além de gerar danos maiores ao bem-estar psicológico, podem interferir na continuidade do tratamento dos pacientes psiquiátricos ao restringir 0 acesso aos medicamentos necessários.

Deve-se ressaltar, ainda, que muitos especialistas têm demonstrado preocupações sobre a contribuição dos problemas mentais, econômicos, psicossociais e comportamentais ligados à pandemia para a elevação dos comportamentos de autoextermínio e automutilação. Nessa ótica, a identificação e o manejo do indivíduo com comportamentos suicidas se tornam ainda mais desafiadores diante da situação de uma pandemia que limita o acesso aos recursos da saúde, além do receio de se contaminar com o vírus. Ademais, deve-se constar que mulheres, jovens e indivíduos de países democráticos são os mais vulneráveis à ideação suicida durante a pandemia (DUBÉ JP, et al., 2021).

Matias T, et al. (2020) apontou que as adaptações decorrentes da COVID-19 cursaram com alterações comportamentais, como o desenvolvimento do hábito de estocar alimentos e outros bens de consumo essenciais, assim como um aumento da necessidade para autoproteção, aliada à diminuição acentuada do contato social por meio de isolamento e quarentenas. Logo, durante a pandemia, ocorreu uma mudança no foco humano e na motivação para as necessidades básicas, que pode explicar a intensidade e seriedade das consequências psicológicas observadas. Dentre as manifestações, podem estar incluídas alterações 
negativas na autoestima e percepção de si, possivelmente afetadas pela crise econômica oriunda da permanência do estado pandêmico, que resultou em perda de empregos, aumento da insegurança econômica e alimentar e seu impacto provável sobre o status social individual.

\section{Tratamento}

Para efeito comparativo, experiências prévias de surtos de infecções virais, como a SARS em 2003 e a MERS (Síndrome Respiratória do Oriente Médio) em 2012, com subsequente isolamento e quarentena, culminaram, por vezes, com distúrbios psicológicos, como ansiedade, episódios depressivos e distúrbios do sono. Dessa forma, cerca de $70 \%$ dos pacientes com diagnóstico de MERS, por exemplo, exibiram sintomas psiquiátricos, enquanto que aproximadamente $40 \%$ receberam algum diagnóstico psiquiátrico definitivo, com uso de medicamentos durante a internação (DENG J, et al., 2021).

Apesar da importância de conduzir corretamente os transtornos psiquiátricos em pacientes com COVID19, ainda não há evidências suficientes para determinar a prevalência destes transtornos. Entretanto sabe-se que esses quadros de ansiedade, depressão e distúrbios do sono, devem ser adequadamente diagnosticados e tratados a fim de melhorar o prognóstico da doença, bem como diminuir o tempo de internação e mitigar problemas de saúde mental no futuro. Sendo assim, muitos indivíduos, em todo o mundo, estão sofrendo os efeitos físicos e mentais da COVID-19 e medidas devem ser tomadas de imediato, sem a necessidade de esperar vários anos, até que dados sólidos estejam disponíveis (DENG J, et al., 2021; LIU D, et al., 2021).

A partir de estudos já conclusivos, pode-se perceber que a maioria dos profissionais da saúde apresentaram sintomas leves de ansiedade e depressão, o que enfatiza a importância de se detectar e tratar esses quadros mais brandos antes que evoluam para quadros mais graves e duradouros. Para isso, muito pode ser oferecido no contexto atual, com clínicas virtuais, terapias psicológicas e psicoeducativas à distância, linhas de bate-papo, monitoramento de tecnologia de alto risco, dentre outros. Sobretudo, pacientes infectados, profissionais de saúde e seus respectivos familiares, que se encontrarem em isolamento domiciliar também devem ser apoiados (PAPPA S, et al., 2020).

Fiorillo A e Gorwood P (2020) salientam a necessidade de orientar a população práticas que podem reduzir os riscos de se desenvolver enfermidades psicológicos, como limitar as fontes de informações que causem estresse e contar com uma quantidade direcionada de fontes oficiais de informações, bem como reduzir 0 tempo do dia dedicado a isso. Ademais, acrescentam que vídeo chamadas com grupos familiares ou com amigos é uma opção para diminuir a solidão do isolamento. Além disso, manter hábitos saudáveis de alimentação, de sono e de atividades intelectuais, ter em mente que este é um período transitório e que o isolamento é benéfico a todos; E por fim, destaca a importância de pedir ajuda a um profissional, por meio de consultas psiquiátricas, à distância, se necessário, quando os efeitos do estresse estiverem intensos.

Assim, pesquisas que analisem os resultados neuropsiquiátricos diretos e os desfechos indiretos na saúde mental são fundamentais para melhorar o tratamento, o planejamento dos cuidados e incentivar ações preventivas durante as possíveis pandemias posteriores (VINDEGAARD N e BENROS NE, 2020). Além disso, esses estudos têm como objetivo incentivar a conscientização sobre a importância do rastreamento e do tratamento de doenças psiquiátricas em pacientes com COVID-19 (DENG J, et al., 2021).

Melnyk BM, et al. (2020), em sua revisão sistemática, observou o efeito de intervenções diversas sobre a saúde de médicos e enfermeiros. Foi identificado maior efeito positivo das terapias baseadas em mindfulness e Terapia Cognitivo-Comportamental (TCC) em relação à qualidade de sono, diminuição de estresse, melhora de humor e de sintomas ansiosos e depressivos. Portanto, os sistemas de saúde mental devem oferecer estratégias desta natureza e adaptar os resultados de estudos para a prática clínica, de modo a promover desfechos favoráveis aos profissionais afetados e consequentemente melhorar a qualidade e segurança do cuidado por eles prestados.

\section{Os desafios para o cuidado com a saúde mental durante e após a pandemia}

Numa situação de difícil crise, a exemplo da pandemia da COVID-19, a preocupação com a saúde mental da população se torna ainda maior. Neste cenário, independentemente de gênero, grupo ou região, os indivíduos são sujeitos a sofrer impactos psicológicos e sociais em vários níveis de intensidade e gravidade, levando a uma alta prevalência de transtornos (WHO, 2018; FIOCRUZ, 2020). 
Segundo Schwartz R et al. (2020), a pandemia da COVID-19 concebeu desafios inéditos devido às incertezas relativas à doença, a percepção de ameaça à segurança pessoal e familiar, a necessidade de isolamento social como forma de evitar o contágio, a experiência de sofrimento físico e morte na "linha de frente" e as demandas profissionais exacerbadas pelo aumento do número de pacientes. Destarte, esses fatores individuais atuam sinergicamente para induzir estados de excitação e vigilância excessivos, distúrbios do sono, pensamentos indesejáveis, luto, sintomas depressivos e ansiosos.

Para Cénat JM, et al. (2021) o grande desafio é oferecer os cuidados e atenção necessários aos indivíduos psiquicamente afetados pelo COVID-19. A implementação de programas de intervenção e prevenção em saúde mental se faz necessária, de forma a tornar os cuidados de saúde o menos traumáticos possíveis para as pessoas que desenvolveram sintomas mentais, graves ou não. Cabe aos profissionais de saúde reconhecerem e abordarem rapidamente os problemas mentais relacionados à pandemia e atuar para evitalos ou minimizar seus impactos a longo prazo.

Em virtude do risco de agravamento da doença de base, pacientes com comorbidades mentais prévias necessitam de maior atenção em saúde mental qualificada e cuidadosa, bem como as populações vulneráveis. Portanto, considerando os efeitos deletérios da pandemia sobre a saúde mental e o bem-estar desses indivíduos, além dos profissionais de saúde e da população em geral, os profissionais de saúde mental precisarão cuidar dos pacientes de forma mais convencional, cautelosa e personalizada (FIORILLO A e GORWOOD P, 2020; SHINN AK e VIRON M, 2020).

Embora os efeitos psicológicos da COVID-19 ainda não tenham sido plenamente esclarecidos em relação ao futuro, é recomendável que os sistemas de saúde se preparem para atender um aumento de demanda por serviços de saúde mental, tendo em vista as taxas de prevalência calculadas em estudos. Sendo assim, a partir das evidências observadas e produzidas durante a pandemia, há a necessidade de identificar as consequências psicológicas de longo prazo, a fim de assegurar a oferta adequada de serviços para todos os acometidos e permitir uma abordagem terapêutica mais efetiva (ARORA T, et al., 2020).

\section{CONSIDERAÇÕES FINAIS}

Mediante este estudo, pôde-se considerar que vários são os fatores que contribuem para o surgimento ou agravamento de transtornos na saúde mental de pacientes envolvidos com a COVID-19. Salienta-se que, tanto pacientes com a doença, quanto os que não a apresentaram, mas tiveram que isolar-se, são mais propensos a terem sua saúde mental prejudicada. Assim, apesar de já terem estudos correlacionando os medicamentos ideais para 0 tratamento desses distúrbios, ressalta-se que terapias cognitivas comportamentais são imprescindíveis. O presente estudo buscou mostrar a necessidade e a importância da implementação de políticas e programas de apoio e atenção aos indivíduos mentalmente acometidos pelos grandes impactos sociais e econômicos, os quais se aplicam, consequentemente, aos impactos psicológicos de toda uma população.

\section{REFERÊNCIAS}

1. ABDALLA SM, et al. Mental health consequences of COVID-19: a nationally representative cross-study of pandemic related stressors and anxiety disorders in the USA. BMJ Open, 2021; 11: e044125.

2. ANAND V, et al. COVID-19 and psychological distress: lessons for India. PLoS One, 2021; 16: e0255683.

3. ARORA T, et al. The prevalence of psychological consequences of COVID-19: a systematic review and meta-analysis of observational studies. Journal of Health Psychology, 2020; 1359105320966639.

4. ASMUNDSON G, et al. Do pre-existing anxiety-related and mood disorders differentially impact COVID-19 stress responses and coping? Journal of Anxiety Disorders, 2020; 74: 102271.

5. BUTTELL F, FERREIRA R. The hidden disaster of COVID-19: Intimate partner violence. Psychological Trauma: Theory, Research, Practice, And Policy, 2020; 12: S197-S198.

6. CASTALDELLI-MAIA JM, et al. Investigating the effect of national government physical distancing measures on depression and anxiety during the COVID-19 pandemic through meta-analysis and meta-regression. Psychological Medicine, 2021; 51: 881-893. 
7. CÉNAT JM, et al. Prevalence of symptoms of depression, anxiety, insomnia, posttraumatic stress disorder, and psychological distress among populations affected by the COVID-19 pandemic: A systematic review and meta-analysis. Psychiatry Research, 2021; 295: 113599.

8. CLAY J, PARKER M. Alcohol use and misuse during the COVID-19 pandemic: a potential public health crisis? The Lancet Public Health, 2020; 5: e259.

9. DENG J, et al., The prevalence of depression, anxiety, and sleep disturbances in COVID-19 patients: a meta-analysis. Annals of New York Academy of Sciences, 2021; 1486: 90-111.

10. DUBÉ JP, et al. Suicide behaviors during the COVID-19 pandemic: A meta-analysis of 54 studies. Psychiatry Research, $2021 ; 301: 113998$.

11. FIORILLO A, GORWOOD P. The consequences of the COVID-19 pandemic on mental health and implications for clinical practice. European Psychiatry, 2020; 63: e32.

12. GOLD JA. COVID-19: adverse mental health outcomes for healthcare workers. BMJ, 2020; 369: m1815.

13. LEE Y, et al. Government response moderates the mental health impact of COVID-19: A systematic review and metaanalysis of depression outcomes across countries. Journal of Affective Disorders, 2021; 290: 365-377.

14. LI Y, et al. Prevalence of depression, anxiety and posttraumatic stress disorder in health care workers during the COVID-19 pandemic: A systematic review and meta-analysis. PLoS One, 2021; 16: e0246454.

15. LIU D, et al. Mental health outcomes of coronavirus infection survivors: A rapid meta-analysis. Journal of Psychiatric Research, 2021; 137: 542-553.

16. MATIAS T, et al. Human needs in COVID-19 isolation. Journal of Health Psychology, 2020; 25: 871-882.

17. MELNYK BM, et al. Interventions to improve mental health, well-being, physical health, and lifestyle behaviors in physicians and nurses: a systematic review. American Journal of Health Promotion, 2020; 34: 929-941.

18. NOCHAIWONG S, et al. Global prevalence of mental health issues among the general population during the coronavirus disease-2019 pandemic: a systematic review and meta-analysis. Scientific Reports, 2021; $11: 10173$.

19. PAPPA S, et al. Prevalence of depression, anxiety, and insomnia among healthcare workers during the COVID-19 pandemic: a systematic review and meta-analysis. Brain, Behavior and Immunity, 2020; 88: 901-907.

20. PAREKH R. What is mental illness?, American Psychiatric Association, 2018. Disponível em: https://www.psychiatry.org/patients-families/what-is-mental-illness. Acessado em 14 de ago. de 2021.

21. PATEL V. Acting early: the key to preventing mental health problems. Journal of the Royal Society of Medicine, 2018; 111: 153-157.

22. PFEFFERBAUM B, NORTH CS. Mental health and the COVID-19 pandemic. New England Journal of Medicine 2020; 383: 510-512.

23. PIETROMONACO P, OVERALL N. Applying relationship science to evaluate how the COVID-19 pandemic may impact couples' relationships. American Psychologist, 2021; 76: 438-450.

24. RAONY I, et al. Psycho-neuro-endocrine-immune interactions in COVID-19: potential impacts on mental health. Frontiers in Immunology, 2020; 11: 1170.

25. SALADINO V, et al. The psychological and social impact of Covid-19: new perspectives of well-being. Frontiers In Psychology, 2020; 11: 2550.

26. SALARI N, et al. Prevalence of stress, anxiety, depression among the general population during the COVID-19 pandemic: a systematic review and meta-analysis. Globalization and Health, 2020; 6: 57.

27. SANTABÁRBARA J, et al. Prevalence of anxiety in the COVID-19 pandemic: An updated meta-analysis of communitybased studies. Progress in Neuropsychopharmacology \& Biological Psychiatry, 2021; 109: 110207.

28. SCHWARTZ R, et al. Addressing post pandemic clinician mental health: a narrative review and conceptual framework. Annals of Internal Medicine, 2020; 173: 981-988.

29. SHER L. Psychiatric disorders and suicide in the COVID-19 era. QJM: An International Journal of Medicine, 2020; 113: $527-528$.

30. SHINN AK, VIRON M. Perspectives on the COVID-19 pandemic and individuals with serious mental illness. Journal of Clinical Psychiatry, 2020; 81: 20com13412.

31. VINDEGAARD N, BENROS ME. COVID-19 pandemic and mental health consequences: systematic review of the current evidence. Brain, Behavior, and Immunity, 2020; 89: 531-542.

32. WANG $L$, et al. Potential neurochemical and neuroendocrine effects of social distancing amidst the COVID-19 pandemic. Frontiers in Endocrinology, 2020; 11: 807.

33. WORLD HEALTH ORGANIZATION (WHO). Mental health: strengthening our response. 2018. Disponível em: https://www.who.int/news-room/fact-sheets/detail/mental-health-strengthening-our-response. Acessado em 20 de maio de 2021.

34. XIANG YT, et al. The COVID-19 outbreak and psychiatric hospitals in China: managing challenges through mental health service reform. International Journal of Biological Sciences, 2020; 16: 1741-1744.

35. ZHU N, et al. A novel coronavirus from patients with pneumonia in China, 2019. New England Journal of Medicine, 2020; 382: 727-733. 